\title{
Multi-colour photometric and spectroscopic monitoring of the WN5 star EZ Canis Majoris ${ }^{\star}$
}

\author{
M.F.J. Duijsens ${ }^{1}$, K.A. van der Hucht ${ }^{2}$, A.M. van Genderen ${ }^{1}$, H.E. Schwarz ${ }^{3}$, H.P.J. Linders ${ }^{1}$ and O.M. \\ $\mathrm{Kolkman}^{4}$ \\ 1 Leiden Observatory, Postbus 9513, NL-2300 RA Leiden, The Netherlands \\ 2 Space Research Organisation Netherlands, Sorbonnelaan 2, NL-3584 CA Utrecht, The Netherlands \\ 3 Nordic Optical Telescope, Apartado 474, E-38700 Sta. Cruz de La Palma, Canarias, Spain \\ 4 Kapteyn Laboratory, Postbus 800, 9700 AV Groningen, The Netherlands
}

Received December 6, 1995; accepted February 21, 1996

\begin{abstract}
We present and analyse photometric and spectroscopic observations of the WN5 star EZ Canis Majoris obtained over a period of 7 years. We discuss the changing light curve, the shift in phase of the maxima and point to flare type variability seen in one night. Small amplitude variations are reported in another night. We have investigated the change of the average visual magnitude over a time span of 18 years and found a tentative cyclic variation with a time scale of $2425^{\mathrm{d}}(6.6 \mathrm{yr})$ with a range of $\sim 0$. 07 . This, of course, should be verified. If true, a precession phenomenon may offer an explanation. The trend of the maximum light amplitude of the 3 . 766 cycle is also investigated and it shows a saw-tooth character with a timescale of $\sim 400^{\mathrm{d}}$. A possible relation with the magnetic activity of the star is discussed. We conclude that the line emission variability can be caused by both a single star model with an ever-changing wind and a binary (WN+NS) model. ${ }^{\star \star}$
\end{abstract}

Key words: stars: Wolf-Rayet — stars: individual: WR $6=$ HD $50896=$ EZ CMa — stars: variable — binaries: close

\section{Introduction}

One of the best studied Wolf-Rayet stars is the apparently brightest single WN 5 star EZ CMa. This object shows a variability that can be interpreted as caused by a binary as well as by a single star. In the latter case the variability could be caused by some type of pulsation, or by a peculiar geometry of the wind. Firmani et al. (1980) concluded that the binary hypothesis is the most likely to explain the periodic variations. They found a period of 3.763. Lamontagne et al. (1986) confirmed this hypothesis and improved the period to 3 d 766 . From X-ray fluxes White \& Long (1986) concluded that the companion of EZ CMa could be a black hole in an eccentric orbit and not a neutron star as thought before. Gosset and Vreux (1987) found indications for a possible second period of almost (but not exactly) one third of the 3.766 period pointing to nonradial pulsations as a possible cause for the light variations. Van der Hucht et al. (1990) in an analysis of a preliminary reduction of the present LTPV

Send offprint requests to: A.M. van Genderen

*Observations were made at the ESO, La Silla, Chile

${ }^{\star \star}$ Tables 7 to 25 are available at the CDS via anonymous ftp 130.79.128.5 data confirmed this period, but found a discontinuity in the variability. This has been discussed and explained by Sterken (1993). An improved reduction available to Gosset et al. (1990) removed this discontinuity. Van der Hucht et al. (1990) also postulated the presence of semi co-rotating clouds with different scattering and absorbing capabilities. Underhill \& Yang (1991) concluded from $12(40 \AA / \mathrm{mm})$ spectra that EZ CMa is a single star with a ring-like, rotating disk connected to the star by a few ever-changing filaments supported by magnetic field lines. The 3.766 period then would represent the rate of rotation of the disk. Schulte-Ladbeck et al. (1991, 1992) interpreted their spectropolarimetric data as the result of a single star with an electron-scattering wind that is axisymmetric, rotating and expanding, with a variable mass loss rate being responsible for the quasi-periodic polarimetric variability. In contrast, Robert et al. (1992) found strong arguments in favour of a binary system. St-Louis et al. (1993) suggested that the ultraviolet spectroscopic variability is intrinsic to the stellar wind and reflects changes in its physical properties. Antokhin et al. (1994) obtained 3 months of continuum photometry and find only one independent significant period, $P=3$ d.766. Marchenko et al. (1994) found evidence for a possible period of $0.11 \mathrm{~s}$, i.e. in the 
expected range for a spin-up pulsar. Howarth \& Schmutz (1995) confirmed that EZ CMa lies beyond the cluster Cr 121 and estimate $d \simeq 1.8 \mathrm{kpc}$. Recently, St-Louis et al. (1995) offered an ad-hoc model in which the star is embedded in some kind of co-rotating interaction region emanating from hot (magnetically?) active regions near the surface of the star.

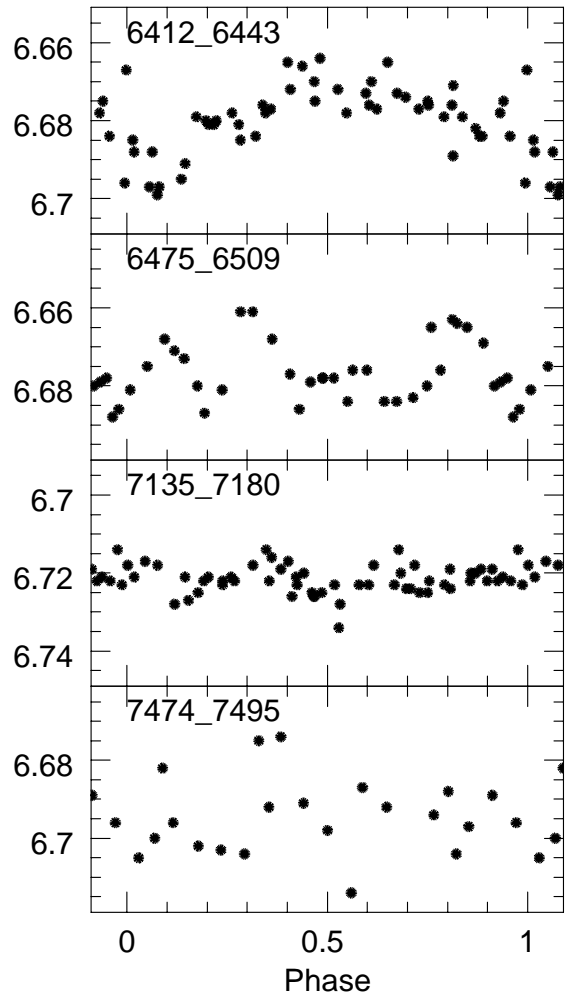

Fig. 1. Phase diagram of Strömgren $y$ magnitude from December 13, 1985 through November 30, 1988, folded with the period of Lamontagne et al. (1986), $2 \sigma<0.01 \mathrm{mag}$

In this paper we present and analyse photometric data obtained over the period of December 1985 through December 1992 and spectroscopic data obtained on December 5 and December 7, 1986.

\section{Telescopes and instruments}

Four telescopes were used for the observations, whereas all the observations were obtained at the ESO (La Silla, Chile). Strömgren photometric observations of EZ CMa were obtained by the LTPV project ("Long-Term Photometry of Variables" Sterken 1983, 1986), with the $0.5 \mathrm{~m}$ Danish telescope during twelve time spans between December 13, 1985 and December 18, 1992. The diaphragm aperture was $17^{\prime \prime}$. Details of the available Strömgren filter systems have been given by Manfroid \& Sterken (1987). Our observations were made with Strömgren filter system No. 7.

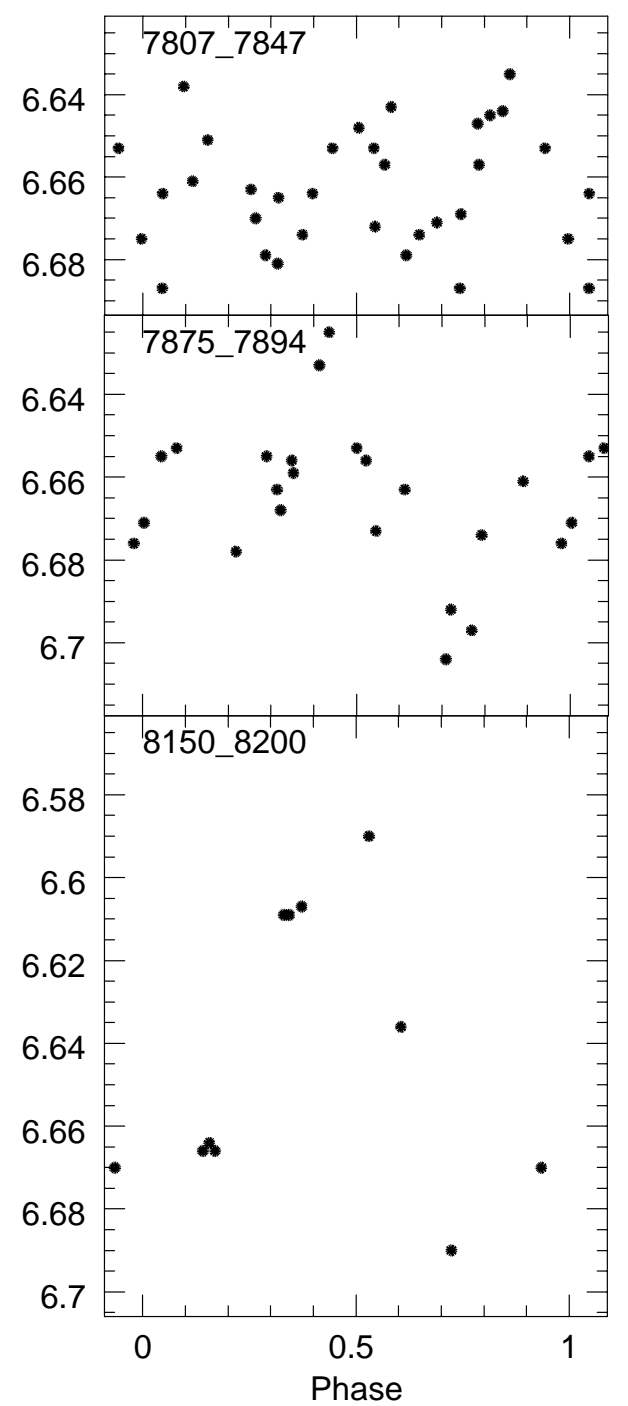

Fig. 2. Phase diagram of Strömgren $y$ magnitude from October 8, 1989 through November 5, 1990, folded with the period of Lamontagne et al. (1986), $2 \sigma<0.01 \mathrm{mag}$

A second set of photometry was obtained with the 0.5 m ESO telescope using the Johnson $U B V$ filter system during January 11, 1987 through January 16, 1987. The diaphragm aperture was $30^{\prime \prime}$.

A third set of photometric observations was obtained with the $0.9 \mathrm{~m}$ Dutch telescope at ESO equipped with the $V B L U W$ photometer of Walraven, during two intervals from November 25, 1988 through December 23, 1988 (epoch I) and December 9, 1989 through December 12, 1989 (epoch II). The diaphragm aperture used was $16 . " 5$. A detailed description of the photometric system is given by Lub and Pel (1977) and references therein.

Spectroscopic observations were obtained with the $1.52 \mathrm{~m}$ ESO telescope, using an Image Dissector Scanner 
(IDS) together with a Boller and Chivens (B\&C) spectrograph. Table 1 lists all observers.

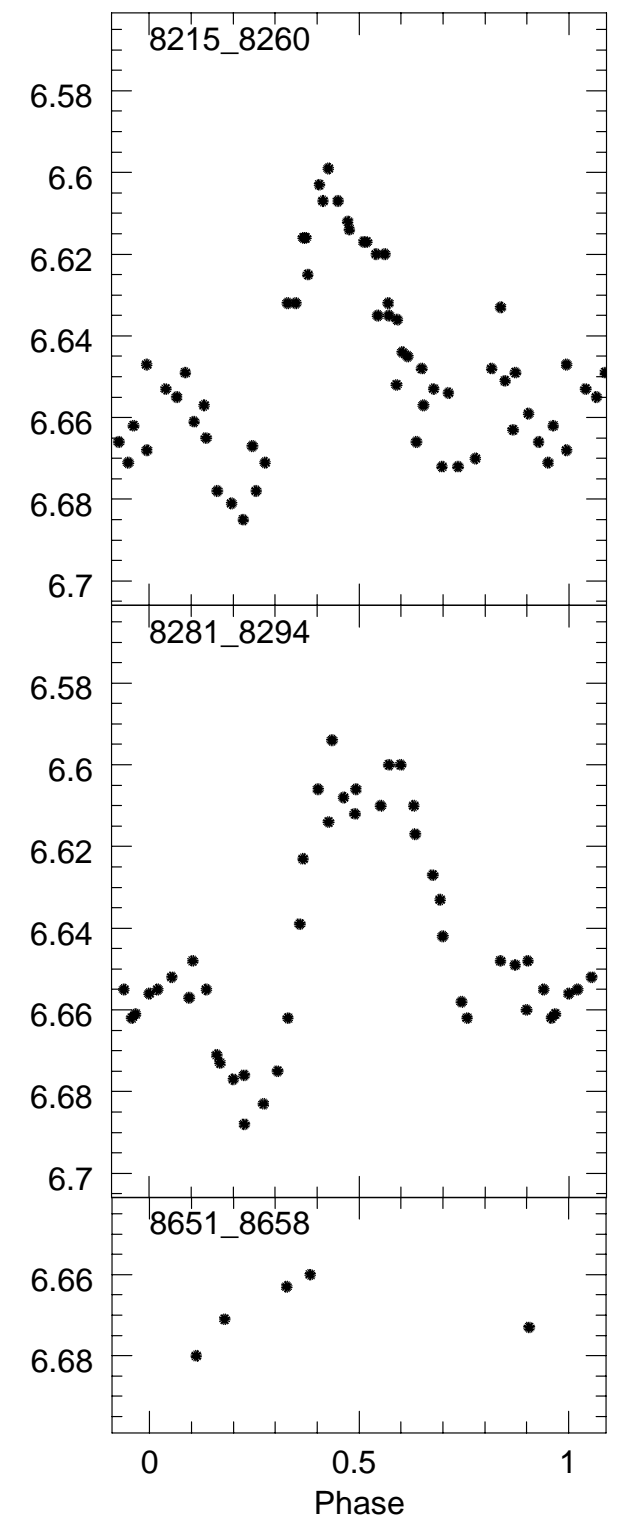

Fig. 3. Phase diagram of Strömgren $y$ magnitude from November 20, 1990 through February 6, 1992, folded with the period of Lamontagne et al. (1986), $2 \sigma<0.01 \mathrm{mag}$

\section{Observations and reductions}

\subsection{Strömgren photometry}

During all of the time spans the comparison stars HD $50853=$ HR 2578, of spectral type A1, HD 50711, of spectral type A2, and HD 50806, of spectral type G5IV were used. The sequence consisted generally out of a few times the program star alternated by the two comparison stars and at the end a sky background. Integration times

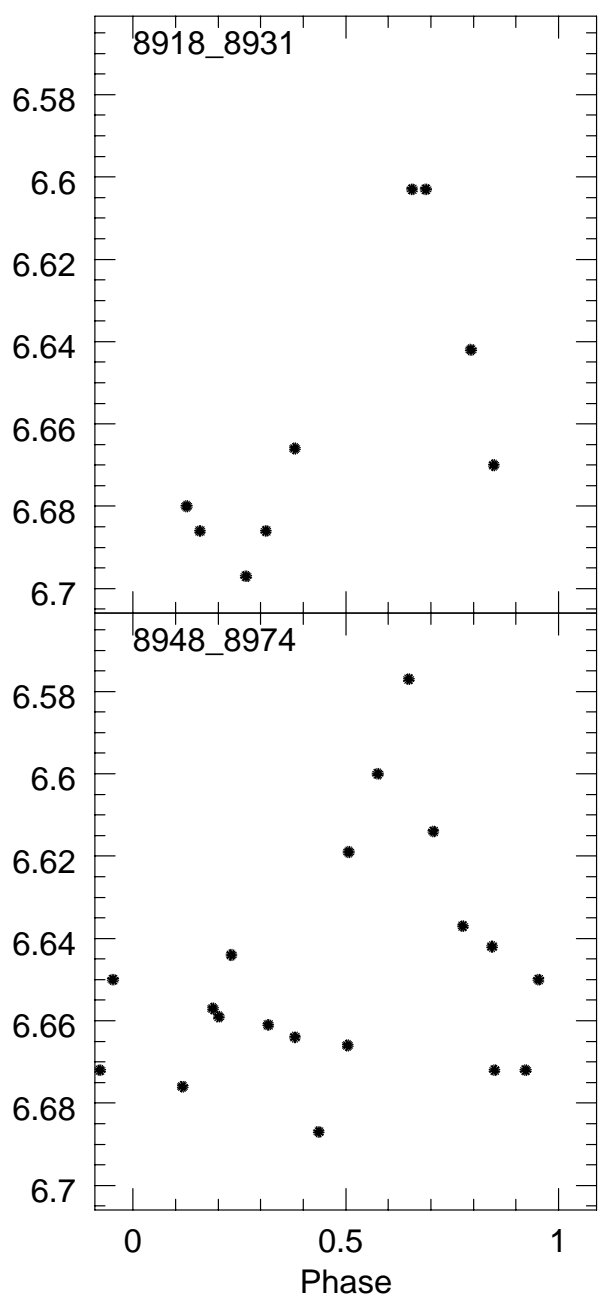

Fig. 4. Phase diagram of Strömgren $y$ magnitude from October 23, 1992 through December 18, 1992, folded with the period of Lamontagne et al. (1986), $2 \sigma<0.01 \mathrm{mag}$

were usually of the order of 1-2 minutes (see also Sterken et al. 1995b). In this paper we shall only discuss the average $y$ magnitudes per night relative to HD 50853. All $y$ observations of the second and third comparison star (called B and C, respectively) were used also by transforming them to the A magnitudes by adding the average value of $\mathrm{A}-\mathrm{B}$ and $\mathrm{A}-\mathrm{C}$, respectively. The individual observations uvby have been published by Manfroid et al. (1991, 1994) and Sterken et al. (1993, 1995b) and will not be given here. Figures 1 through 4 show phase diagrams for the $y$ magnitudes only of all epochs, from December 13, 1985 through December 18, 1992, where JD-2.440.000 of the first and last observation is given in the upper left corner of each phase diagram. The first two panels in Fig. 1 concern $y$ observations of which also the colour variations are discussed in detail by van der Hucht et al. (1990). Also for the other data sets colour variations could be established, but will not be discussed here. As in all cases in this paper all data are folded with the period of Lamontagne 
et al. (1986): $P=3$ d 766 . The error in the observations is at most $0.005 \mathrm{mag}$ and usually smaller. Table 2 lists the photometric parameters of the comparison stars in the Strömgren system.

\subsection{High-time resolution Johnson photometry}

For the observing period in January 1987 two comparison stars were used: C1 (HR $2598=$ HD 51411, B3V) and C2 $($ HR $2607=$ HD 51733, F3V). EZ CMa was observed in the sequence C1-EZ-C2-EZ with at the end a sky background. Integration times were usually in the order of 1-2 minutes. C2 was used to calculate the extinction coefficients of all nights. Although it is better to use a comparison star with a spectrum close to that of $\mathrm{EZ} \mathrm{CMa,} \mathrm{we} \mathrm{used} \mathrm{C} 2$, the $\mathrm{F}$ type star, since for $\mathrm{C} 1$ no $U$ magnitude was available. We checked the effect of atmospheric reddening and found no significant difference between the extinction coefficients calculated with $\mathrm{C} 1$ and $\mathrm{C} 2$. Figure 5 shows Johnson $V$ magnitude for EZ CMa from January 11, 1987 through January 16, 1987. Table 2 lists the photometric parameters of the comparison stars in the Johnson system. Tables 7 through 12 (available electronically) list the differential magnitudes and colours and can be obtained in electronic form.

\subsection{Walraven photometry}

The comparison star used during the two observational runs (epochs I and II) was also HD 50853, of spectral type A1, as for the Strömgren photometry in Sect. 3.1 and for the Walraven photometry made in 1986 (van Genderen et al. 1987). The $V B L U W$ photometric parameters derived from the comparison with standard stars are separately listed for both epochs (Table 2). The differences are small enough to conclude that the star was constant. The regular observing routine was to measure EZ CMa four times alternated by the comparison star. The integration times were usually 1-2 minutes. However, in the nights of 10 , 11, 12 and 14 December 1988, EZ CMa was measured about 24 times after another followed by a measurement of the comparison star. This explains the increased noise for these four nights. The $V$ and $(B-V)$ magnitudes of the Johnson $U B V$ system (with subscript J) can be obtained from the equivalent Walraven $V$ and $V-B$ with the formulae of Pel (1985):

$$
V_{\mathrm{J}}=6.885-2.5[V+0.030(V-B)]
$$

and

$$
\begin{gathered}
(B-V)_{\mathrm{J}}=2.571(V-B)-1.020(V-B)^{2} \\
+0.500(V-B)^{3}-0.010 .
\end{gathered}
$$

Figure 6 shows the differential Walraven $V$ phase diagram from all 1988 observations. Figures 7 through 9 show the results of the monitoring nights in 1988 and 1989 in detail.
In Fig. 8 the results of the comparison star are shown because it then is obvious that any variation in the flux of EZ CMa on a time scale longer than about $20^{\mathrm{m}}\left(0.014^{\mathrm{d}}\right)$ is real. An error bar is therefore not necessary. The variations of the comparison star $c(\sim 1.5 \%)$ are not real, but due to slight variations in the atmospheric extinction. Tables 13 through 25 (available electronically) list the differential $V B L U W$ brightnesses and can be obtained in electronic form.

\subsection{IDS spectroscopy}

On December 5 and 7, 1986, 44 IDS spectra of EZ CMa were obtained with the Boller \& Chivens spectrograph on the $1.52 \mathrm{~m}$ ESO telescope (Table 3). The Image Dissector Scanner (IDS) is a steerable position sensitive photomultiplier. The photons pass through a proximity-focussed intensifier (3 Varo-tubes) and fall upon a phosphor layer where the image is scanned. With a small slit any two dimensional stellar image will give a spectral image that looks like a gaussian ridge. With these observations the scanner was not scanning parallel to this ridge, so the result is that the 'flat field' is a part of a gaussian. We corrected for this and for the well known non-linearity of the IDS (Rybski 1980; Rosa 1985). The observed spectrum can then be reduced to the real stellar spectrum, applying:

$$
\mathrm{O}=(\mathrm{R} \cdot \mathrm{E} \cdot \mathrm{S})^{1.04} \cdot \mathrm{G}
$$

with $\mathrm{O}$ the observed spectrum, $\mathrm{R}$ the wavelength response of the IDS, E the extinction, $\mathrm{S}$ the real stellar spectrum and $\mathrm{G}$ the gaussian flat field.

In the night of December 5, 33 IDS spectra were taken with integration times between $5^{\mathrm{m}} 18^{\mathrm{s}}$ and $14^{\mathrm{m}} 27^{\mathrm{s}}$ with an average of $10^{\mathrm{m}} 50^{\mathrm{s}}$. In the night of December 7, 11 IDS spectra were taken with integration times between $10^{\mathrm{m}} 38^{\mathrm{s}}$ and $22^{\mathrm{m}} 51^{\mathrm{s}}$ with an average of $12^{\mathrm{m}} 10^{\mathrm{s}}$. Figure 10 shows an example of a spectrum with an integration time of $10^{\mathrm{m}} 50^{\mathrm{s}}$ taken in the first night at 4:32 UT.

\section{Analysis and discussion}

\subsection{Short time-scale photometry}

The interpretation of the light variability of EZ CMa is difficult as is obvious from Figs. 1 through 4, showing the phase diagrams of Strömgren $y$ over a period of 7 years. Note that the contribution of line emission to Strömgren $y$ is relatively larger than for Johnson $V$ (see Table 5). Consequently $y$ is not comparable to the $V_{\mathrm{J}}$ (as it should) directly obtained with an $U B V$ photometer (see the $V_{\mathrm{J}}$ lightcurves in Fig. 5). In Fig. 1 the amplitude of the variations is generally smaller than in Figs. 2 through 4, also the number of maxima per cycle is changing on a long timescale. For example from October 1989 through December 1992 one can see three maxima in the Strömgren 

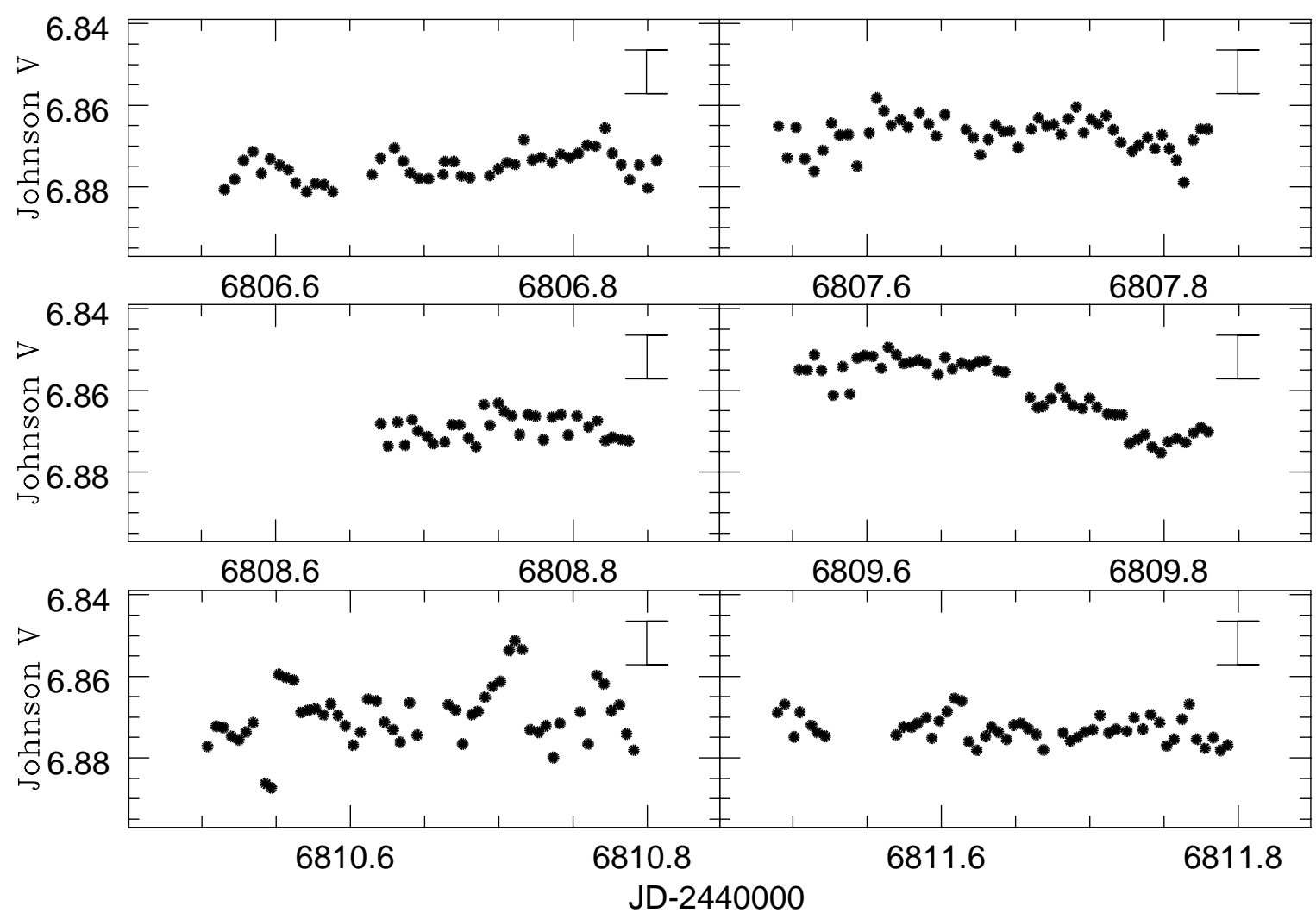

Fig. 5. Johnson $V$ magnitude for EZ CMa from January 11, 1987 through January 16, 1987; the errorbar is $2 \sigma$ large. Typical time interval is 10 minutes

Table 1. List of observers

\begin{tabular}{|c|c|c|c|c|c|}
\hline Date & & JD-2440000 & Observer & Phot./Sp. system & Monitoring \\
\hline Dec.13 1985 & - Jan.13 1986 & $6412-6443$ & F.-J. Zickgraf & Strömgren $u v b y$ no.7 & \\
\hline Feb.14 1986 & - Mar. 71986 & $6475-6496$ & M. Burger & Strömgren uvby no.7 & \\
\hline Mar. 91986 & - Mar.20 1986 & $6498-6509$ & A. Jorissen & Strömgren $u v b y$ no.7 & \\
\hline Dec. 51986 & - Dec. 71986 & $6769-6771$ & H.E. Schwarz & IDS spectroscopy & 2 nights \\
\hline Jan.11 1987 & - Jan.16 1987 & $6806-6811$ & H.E. Schwarz & Johnson UBV & 6 nights \\
\hline Dec. 61987 & - Jan.20 1988 & $7135-7180$ & Y.K. Ng, E. Bibo & Strömgren uvby no.7 & \\
\hline Nov. 91988 & - Nov.30 1988 & $7474-7495$ & M. Hiesgen & Strömgren $u v b y$ no.7 & \\
\hline Nov.25 1988 & - Dec.23 1988 & $7490-7518$ & H.P.J. Linders & Walraven $V B L U W$ & 4 nights \\
\hline Oct. 81989 & - Nov.17 1989 & $7807-7847$ & A. Barzewski, A. Juettner & Strömgren $u v b y$ no.7 & \\
\hline Dec. 91989 & - Dec.12 1989 & $7869-7872$ & O.M. Kolkman & Walraven $V B L U W$ & 4 nights \\
\hline Dec.15 1989 & - Jan. 31990 & $7875-7894$ & M. Püttmann & Strömgren $u v b y$ no.7 & \\
\hline Sep.16 1990 & - Oct. 11990 & $8150-8165$ & R. van Dijk & Strömgren uvby no.7 & \\
\hline Oct. 21990 & - Oct. 91990 & $8166-8173$ & N. Vogt & Strömgren $u v b y$ no.7 & \\
\hline Oct.21 1990 & - Nov. 51990 & $8185-8200$ & R. Kneer & Strömgren uvby no.7 & \\
\hline Nov.20 1990 & - Dec.14 1990 & $8215-8239$ & B. Cunow & Strömgren $u v b y$ no.7 & \\
\hline Dec.19 1990 & - Jan. 41991 & $8244-8260$ & M. Niehues & Strömgren $u v b y$ no.7 & \\
\hline Jan.25 1991 & - Feb. 71991 & $8281-8294$ & A. Jorissen & Strömgren $u v b y$ no.7 & \\
\hline Jan.30 1992 & - Feb. 61992 & $8651-8658$ & M. de Groot & Strömgren uvby no.7 & \\
\hline Oct.23 1992 & - Nov. 51992 & $8918-8931$ & D. Beele & Strömgren $u v b y$ no.7 & \\
\hline Nov.22 1992 & - Dec.18 1992 & $8948-8974$ & K. Goecking & Strömgren $u v b y$ no.7 & \\
\hline
\end{tabular}




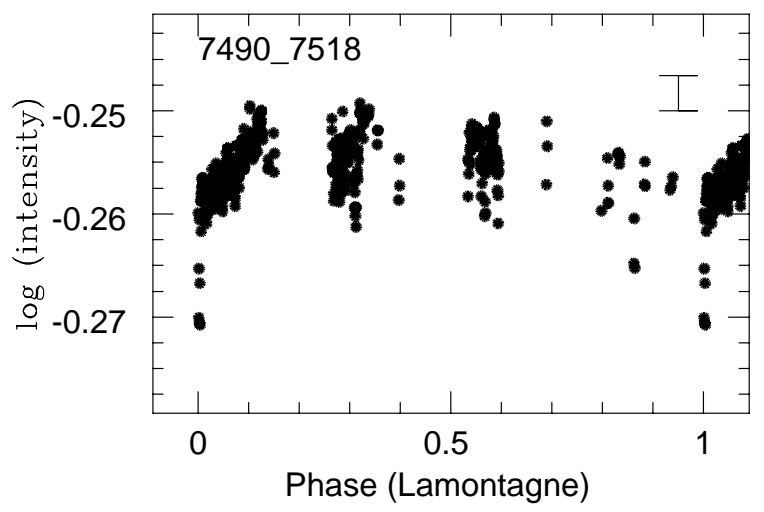

Fig. 6. Phase diagram of differential Walraven $V$ from November 25, 1988 through December 23, 1988; the errorbar is $2 \sigma$ large

$y$ phase diagrams at phase $0.1,0.5$ and 0.85 (Figs. 2-4). The second maximum grows in amplitude from 0.02 to 0. 045 and develops a 'shoulder' in December 1990 and January 1991 at phase 0.55 . This shoulder shifts to phase 0.65 at the end of 1992 (Fig. 4).

In the night of January 15, 1987 we observed a flare type variability in all Johnson passbands at JD 2.446.810.55 and JD 2.446.810.7 (Fig. 5). In the Johnson $V$ magnitude the amplitudes were of the order of 0.026 magnitude $(5 \sigma)$ and the duration was of the order of one hour. These variations may be of the same type as the flare seen by Matthews et al. (1992) on December 1, 1991. However, their flare was of shorter duration $(\approx 10 \mathrm{~min}$.) and of smaller amplitude $(\approx 0.008 \mathrm{mag})$.

In the night of December 11, 1988 small amplitude oscillations $(3 \sigma)$ are visible in Walraven $V$ (Fig. 7) and also in Walraven $B, L, U$ and $W$. However, we need to emphasise that the comparison star was not observed as often as the program star. Therefore we are not quite sure that the observed oscillations are intrinsic to the star.

In the nights of December 11 and 12, 1989 there is a remarkable difference between the trends of the brightnesses in all $V B L U W$ passbands pointing to a contribution of line emission variability (Fig. 9). While, between JD 2.447.671.55 and JD 2.447.671.7, the fluxes in the $V$ and $B$ passbands are weakening, the fluxes in the $L$ and $W$ passbands are brightening. Between JD 2.447.671.7 and JD 2.447.671.85 all brightnesses behave similarly. Between JD 2.447.672.55 and JD 2.447.672.7 the fluxes in the $V$ and $B$ passbands are almost constant, where the fluxes in the $L$ and $W$ passbands are brightening. A possible explanation for this difference is that the light variations are partly caused by continuum variations and partly by emission line variations.
We conclude from the Strömgren photometry plotted in the phase diagrams $(\sim 300$ cycles, Figs. $2-4)$ that the light curves show a more or less fixed structure in which one peak dominates and slowly shifts from phase 0.45 to 0.6. In Fig. 1 this peak may be identified at phase 0.3-0.4. It looks as if the source of this peak (and presumably of all peaks) is somewhat lagging behind and not rigidly rotating with the star (or with the binary). This could fit in the model of St-Louis et al. (1995) which consists of a co-rotating structured wind pattern. However, see Sect. 5. According to the shift of the highest peak discussed above, this pattern is obviously lagging behind.

We further conclude that the behaviour of the different Walraven brightnesses in the nights of December 11 and 12, 1989 clearly points to line emission variability of HeII and NIV. Further investigations of this kind can possibly shed some light on the stratification of the wind.

\subsection{Long time-scale photometry}

We also investigated the changes of the average visual magnitude per data set and the maximum amplitude of the 3.766 cycle over a period of 18 years. Table 4 lists the observation interval per data set, the average magnitude with an uncertainty of 0.02 (see further), the maximum light amplitude, the photometric system or $\lambda_{\text {eff }}$ of the narrow band photometry and the reference.

The upper panel in Fig. 11 shows all the individual Strömgren $y$ and Walraven $V$ magnitudes as a function of time. The latter were transformed to $V_{\mathrm{J}}$. In one week EZ CMa was observed in both systems simultaneously. It appeared that the Walraven $V_{\mathrm{J}}$ was systematically fainter by $0 .{ }^{\mathrm{m}} 182$. This is a consequence of the fact that for emission line objects the transformation formulae are not always quite applicable. Consequently, all other Walraven $V_{\mathrm{J}}$ magnitudes were brightened by that amount.

The lower panel in Fig. 11 shows all photometry, now including the $V_{\mathrm{J}}$ magnitudes based on observations with an $U B V$ photometer. It appeared that these $V_{\mathrm{J}}$ magnitudes and the $y$ magnitudes also differed by $\sim 0$. 182 . Evidently, Fig. 11 shows a long timescale variation.

In the upper panel of Fig. 12 the average $V_{\mathrm{J}}$ (Johnson) magnitudes were corrected for the difference of $-0{ }^{\mathrm{m}} 182$. Data sets at JD 2.444.592 and 2.448.655 are only based on a few observations. The search for a possible cyclic variation was made with the aid of the period search program of Sterken (1977). The search was made between $1700^{\mathrm{d}}$ and $3700^{\mathrm{d}}$ with steps of $50^{\mathrm{d}}$ for $y, V-0^{\mathrm{m}} 182$ and $V_{\mathrm{J}}-0^{\mathrm{m}} 182$. We found as best period $2425^{\mathrm{d}}(6.6 \mathrm{yr})$, the arbitrary zero-point is JD 2.444.357.5. The estimated uncertainty is $\pm 150^{\mathrm{d}}$. The phase diagram in Fig. 13 shows an obvious curve with an amplitude of 0.07 . However, only two cycles are covered, so further monitoring is needed to 


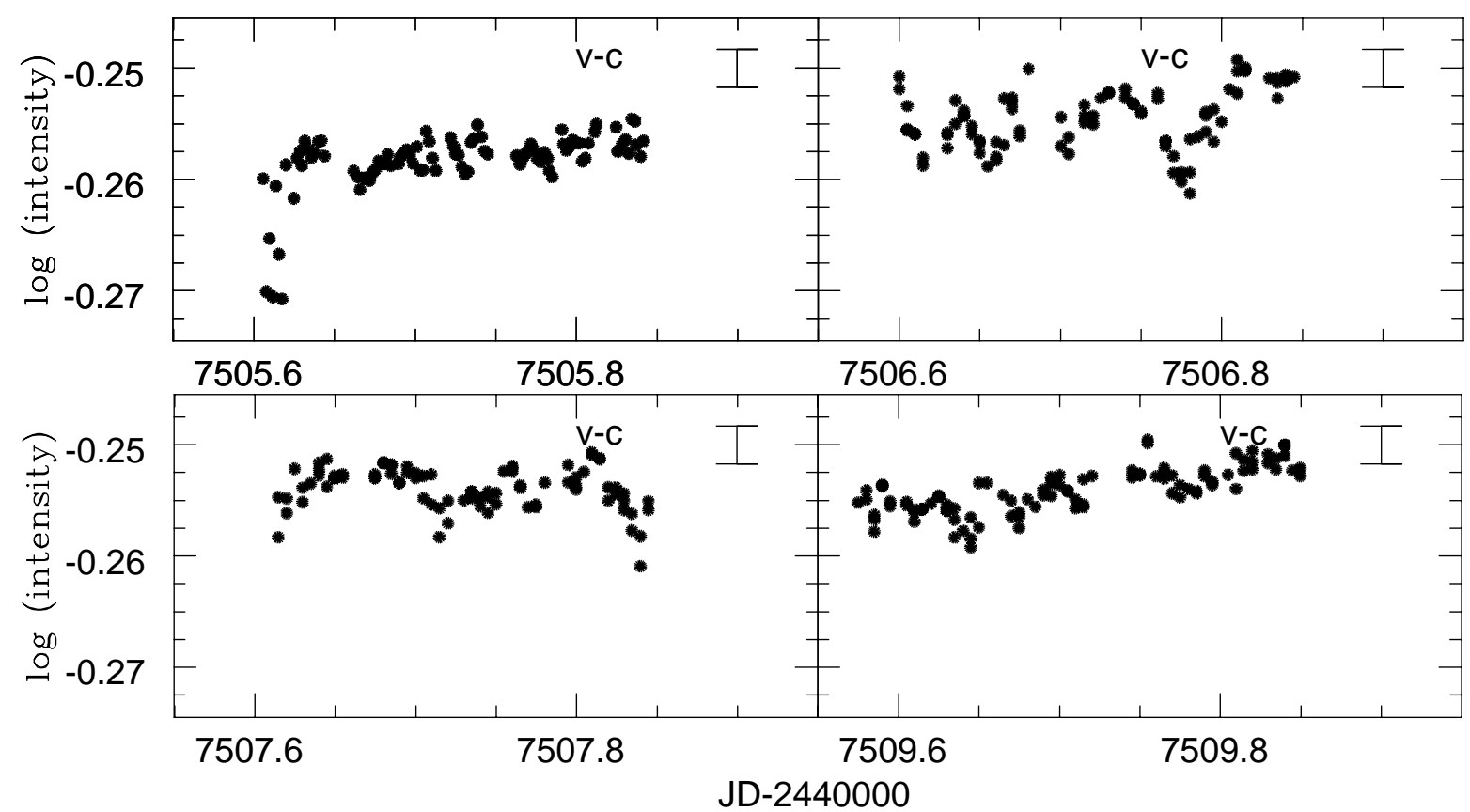

Fig. 7. Differential Walraven $V$ from December 10, 1988 through December 14, 1988; the errorbar is $2 \sigma$ large. 0.01 in $\log ($ intensity $)$ scale $=0.025$

establish the significancy of the periodicity.

In the lower part of Fig. 12 the maximum light amplitude of the 3.766 cycle in $y, V, V_{\mathrm{J}}$ and in the narrow band filters are plotted versus time. The $y$ and $V$ are plotted as filled dots, the $V_{\mathrm{J}}$ as circles and the crosses are the narrow band amplitudes. There is no correlation between the average magnitude and the amplitude, after JD 2.446.000 connected by lines. The narrow band filters exclude the emission lines so they may give different amplitudes than the medium and broad band filters in the visual. A search for a possible cyclic behaviour, with the same program as above, was made between $300^{\mathrm{d}}$ and $700^{\mathrm{d}}$ with steps of $5^{\mathrm{d}}$ excluding the narrow band filters and the data sets based on a few observations. The result was much less convincing than for the average magnitude; the best period was $390^{\mathrm{d}}$ with a correlation coefficient $r=0.61$ and an estimated uncertainty of $\pm 20^{\mathrm{d}}$. It would also mean that around JD 2.448.000 the amplitudes should have been low again (Fig. 12). We tentatively assumed that the difference between the light amplitudes as measured through the $y$, $V$ and $V_{\mathrm{J}}$ filters is negligable.

At first sight the cause of the possible cyclic variation of the mean magnitude may be a precessing luminous disk around the star. When the system is brighter than average, the disk is seen at such an inclination angle that the projected surface of the disk is larger than when the system is fainter than average. However, one then would expect a curve more like a sine wave. Instead of that, the minimum is narrow and the maximum is broad. The long-term variation of the colours is derived from the Strömgren photometry alone. These $u, v, b$ curves (not shown) look similar to that for $y$ in Fig. 12, with the same scatter, but with different amplitudes. The total variation in the four passbands is as follows: $\Delta u=0^{\mathrm{m}} 12, \Delta v=0 \cdot^{\mathrm{m}} 16, \Delta b=0 \cdot^{\mathrm{m}} 04$ and $\Delta y=0.07$. The increasing amplitude to the ultraviolet, equivalent to more than $10 \%$ of the flux, suggests that continuum variations by temperature variations are likely the cause of the $6.6 \mathrm{yr}$ periodicity. Since the $b$ band contains a $67 \%$ contribution of emission lines (Table 5), the amplitude is evidently suppressed. Apart from this fixed influence in all passbands, we are not sure whether the variability of the emission lines can be ignored and whether it is independent of the 6.6 yr periodicity. Consequently, a more precise quantitive interpretation of these amplitudes cannot be offered here.

Together with the fact that the $6.6 \mathrm{yr}$ periodicity is not a symmetric sine wave, the progressive increase of the light amplitudes to the ultraviolet is perhaps not in favour of a precessing luminous disk as it was at first sight (see above). A precessing WR star could be another option: during the precesion cycle we face the star, of which the surface may not be uniformly bright, from various viewing angles. Besides, the star is presumably embedded in a disk-like structured wind pattern (St-Louis et al. 1995) which, dependent on the thickness, then can act as a variable blanketing layer. However, precession of a star means that it is deformed by a nearby companion, unless only the 


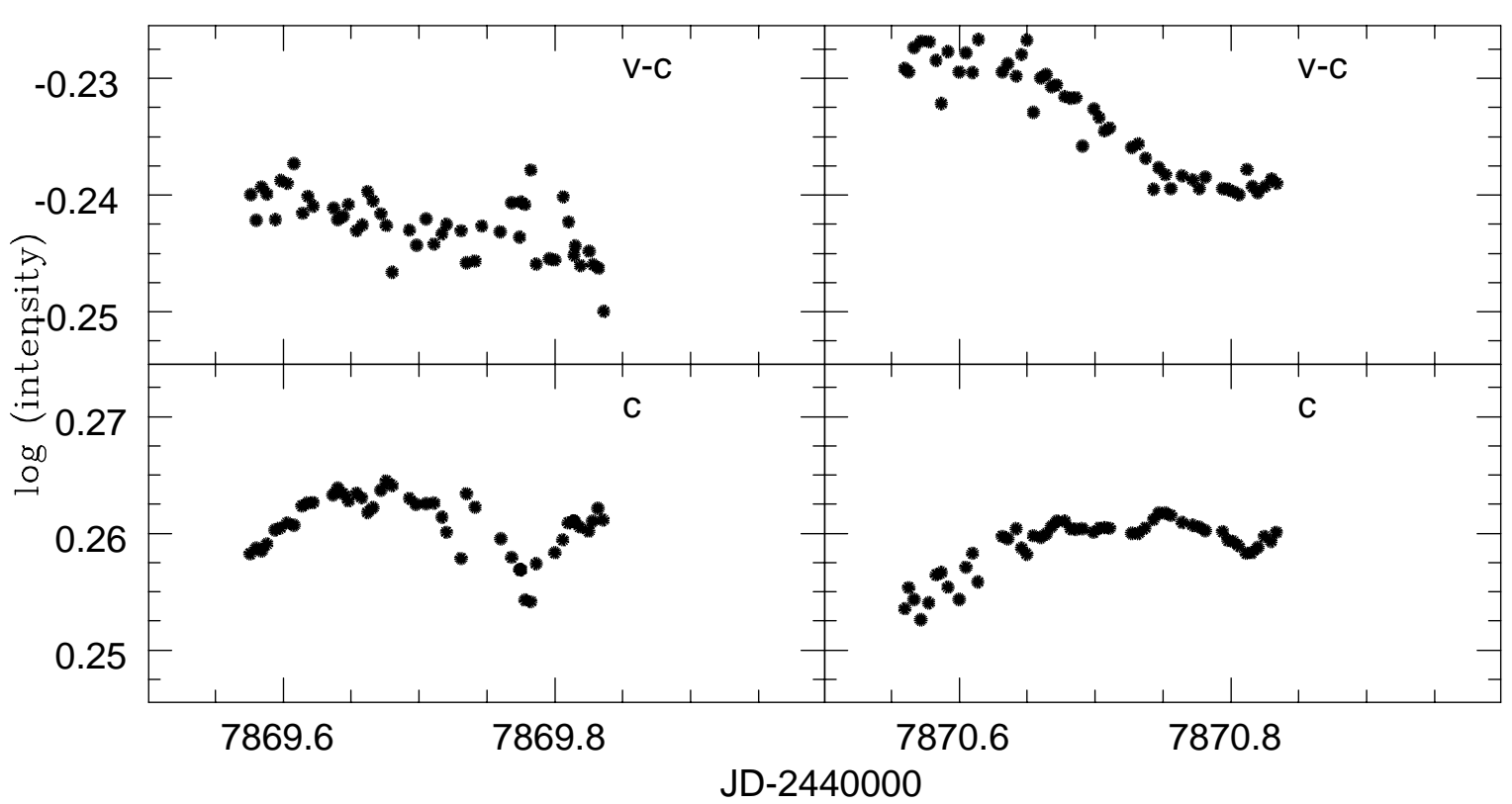

Fig. 8. Differential Walraven $V$ on December 9, 1989 and December 10, 1989 for EZ CMa $(v-c)$ (upper panels) and the trend of the absolute $V$ brightness of the comparison star HD 50853 (c)(lower panels). 0.01 in $\log ($ intensity $)$ scale $=0$. 025

structured disk-like wind pattern is precessing because of some reason.

\subsection{Walraven $V$ and $B$ photometry vs. IUE-FES}

Of further interest is the comparison of our observations from JD 2.447.505 through JD 2.447.510 and the partly simultaneously obtained optical magnitudes estimated from IUE's guidance \& acquisition system's fine error sensor (FES) of St-Louis et al. (1993) (Fig. 14). Note that our Walraven $V$ and $B$ filters are in the same wavelength region as their FES filter that has a response which extends between 4000-7000 $\AA$. Apart from the difference in mean magnitude there is a large discrepancy between our light curves, where the Walraven $V$ brightness is transformed to $V_{\mathrm{J}}$ (uncorrected), and those of St-Louis et al. While our light curves run nearly horizontally with a very small scatter, their light curves show a rather steep rising trend. As an example we show in Fig. 15 the more detailed light curves (Walraven $V$ and $B$, and FES) of JD 2.447.506. Unless the variation of the FES magnitudes is exclusively due to the red part of the FES filter, which lies outside the $V$ filter (thus between $6000 \AA$ and $7000 \AA$ ), we feel puzzled about the reality of the oscillating character of the FES data which, according to St-Louis et al. (1993), show a recurrence time scale of $\sim 1^{\mathrm{d}}$. It is conceivable that some reduction of the amplitude of variation in the Walraven $V$ and $B$ filters has occured due to dilution of continuum light by the emission lines, but the contribution is not that large: $11 \%$ and $22 \%$, respectively (Table 5) and comparable to that of the FES filter (20\%).

\subsection{Short time-scale spectroscopy}

To get more insight into the physical processes that cause the photometric flux variations we estimated how large the line emission contributions are in the three filter systems. We used the atlas of Wolf-Rayet line profiles of Smith \& Kuhi (1981) and approximated the continuum flux by means of a logarithmic function. We sampled their spectrum of EZ CMa every $10 \AA$, convolved it with the transmission curves of each filter system, and calculated the ratio of line emission contribution to the total flux. These results are listed in Table 5 . The identifications of the various lines are listed in Table 6.

We could not detect real emission line variation within a night, although we noted $1 \sigma$ variations in two nights. There are various reports that they do occur in EZ CMa e.g. Ebbets (1979), St-Louis (1994) and Smith \& Willis (1994). We did find a variation in the nightly means: in Fig. 16 one can see a bump on the right shoulder of all four lines in the night of December 5, where it has disappeared in the night of December 7. The profiles are more or less similar to the synthetic profiles of Koenigsberger \& Auer (1992) and Koenigsberger (1995) where they illustrated the effects on the profiles due to a physical eclipse and the contribution by line emission from a companion. In Fig. 17 an observation of the HeII line at $4200 \AA$ is compared with a synthetic HeII line from Fig. 2 of Koenigsberger (1995). However, this feature can also be explained by a 
single star with a rotating, expanding model envelope with bipolar outflows (Matthews et al. 1992).

\section{Summary and conclusion}

We have presented extensive multi-colour photometry and some spectroscopic monitoring of EZ CMa (WR $6=$ HD 50986). According to the long runs of Strömgren photometry, the amplitude of the light variations gradually grew and the number of maxima changed on a time scale of a year or so. We found a long-term shift in the phase of the maxima in the light curves. It could fit the model of St-Louis et al. (1995) that the maxima are caused by places in the co-rotating structured wind with a better transparency. Apparently, this structured wind pattern, is somewhat lagging behind: in 660 cycles the highest peak shifts from phase 0.3 to 0.6 . In 1991 one of the maxima developed a 'shoulder' that subsequently shifted in phase. The cause may be sought in changes of the low and high transparency regions.

The Johnson photometry collected in 1987 shows possible flares, may be of the same type as the flare detected by Matthews et al. (1992), however, ours were longer in duration $\left(\sim 1^{\mathrm{h}}\right)$ and larger in amplitude $(\sim 0.03)$. In 1988 small-amplitude variations are visible in all five Walraven passbands and in 1989 the observations in the same photometric system suggest that continuum and line emission variations occur at the same time. According to the spectroscopic monitoring in December 1986 line emission variation can occur on a time scale of a day.

The long-term trends of the mean brightness and the maximum light amplitude are demonstrated in Figs. 1113 . The first shows possibly a cyclic variation of 6.6 years which certainly should be verified and a range of $0{ }^{\mathrm{m}} 07$ in the visual magnitude. A precessing WR star could offer an explanation: during the precession cycle the star, perhaps embedded in a disk-like nearly co-rotating structured wind, can then be seen from various viewing angles. The light amplitude varies in a saw-tooth manner, with a time scale of $\sim 400^{\mathrm{d}}$, but a strict periodicity could not be established.

To the hypothesis that the nearly co-rotating inhomogeneous density distribution in the wind causing varying transparency could emanate from hot magnetically active regions near the surface of the star (St-Louis et al. 1995), we could add the possibility that the $400^{\mathrm{d}}$ time scale for the 3.766 - maximum light amplitude variation is caused by the magnetic cycle of the star. When the star is magnetically less active, the wind pattern disappears largely and the light amplitude in the phase diagram has no, or only little, peaks. A very low amplitude single wave curve with a number of hardly detectable secondary bumps could then be the result, see e.g. the light and colour curves in Fig. 3a of van der Hucht et al. (1990) (the light curve is also shown in the upper panel of Fig. 1). Thus, the wind pattern should not be always present and might then be modulated with the $\sim 400^{\mathrm{d}}$ time scale of the light amplitude variations. This can only be verified by simultaneous photometric and spectroscopic observations.

Although there now is a vast amount of broad and medium broad band photometry available, the lack of enough spectroscopic monitoring simultaneous with narrow band continuum photometry, is the cause that we cannot distinguish between the single star model with an ever-changing wind and the binary $(\mathrm{WN}+\mathrm{NS})$ model. However, there may be some support for the latter in view of the double peak in the four prominent emission lines in one night (December 5, 1986) and their disappearance two nights later (December 7, 1986).

Acknowledgements. Thanks to a diagram of $y$ magnitudes (from the LTPV project alone) versus time, sent to us by C. Sterken, we decided to disentangle the results of the various photometric systems, revealing a better result on the longterm variations. We are much indebted to referee Dr. Nicole St-Louis for her invaluable remarks.

\section{References}

Antokhin I., Bertrand J.-F., Lamontagne R., et al., 1994, AJ 107,2179

Cherepashchuk A.M., 1981, MNRAS 194, 755

Drissen L., Robert C., Lamontagne R., et al., 1989, ApJ 343, 426

Ebbets 1979, PASP 91, 804

Firmani C., Koenigsberger G., Bisiacchi G.F., et al., 1980, ApJ 239,607

van Genderen A.M., van der Hucht K.A., Steemers W.J.G., 1987, A\&A 185, 131

Gosset E., Vreux J.-M., 1987, A\&A 178, 153

Gosset E., Vreux J.-M., Manfroid J., et al., 1990, A\&AS 84, 377

Howarth I.D., Schmutz W., 1995, A\&A 294, 529

van der Hucht K.A., van Genderen A.M., Bakker P.R., 1990, A\&A 228, 108

Koenigsberger G., Auer L.H., 1992, in: Drissen L., Leitherer C., Nota A. (eds.), Nonisotropic and Variable Outflows from Stars, ASP Conf. Ser. 22, 239

Koenigsberger G., 1995, in: van der Hucht K.A., Williams P.M. (eds.), Wolf-Rayet Stars: Binaries, Colliding Winds, Evolution, Proc. IAU Symp. 163, 538

Lamontagne R., Moffat A.F.J., Lamarre A., 1986, AJ 91, 925

Lub J., Pel J.W., 1977, A\&A 54, 137

Manfroid J., Sterken C., 1987, A\&AS 71, 539

Manfroid J., Sterken C., Bruch A., et al., 1991, First Catalogue of Stars Measured in the Long-Term Photometry of Variables Project (1982-1986), ESO Sci. Rep. No. 8 
Manfroid J., Sterken C., Cunow B., et al., 1994, Third Catalogue of Stars Measured in the Long-Term Photometry of Variables Project (1990-1992), ESO Sci. Rep. No. 14

Marchenko S.V., Antokhin I., Bertrand J.F., et al., 1994, AJ 108,678

Matthews J.M., Moffat A.F.J., Marchenko S.V., 1992, A\&A 266, 409

Pel J.W., 1985, internal report, Leiden Observatory

Robert C., Moffat A.F.J., Drissen L., et al., 1992, ApJ 397, 277

Rosa M., 1985, The Messenger 39, 15

Rybski P.M., 1980, BAAS 12, 751

Schulte-Ladbeck R.E., Nordsieck K.H., Taylor M., et al., 1991, ApJ 382, 301

Schulte-Ladbeck R.E., Nordsieck K.H., Code A.D., et al., 1992, ApJ 391, L37

Smith L.F., Kuhi L.V., 1981, JILA Rep. \# 117

Smith L.F., Willis, 1994, Ap. Sp. Sc. 221, 189

Sterken C., 1977, A\&A 57, 361

Sterken C., 1983, The Messenger 33, 10
Sterken C. 1986, in: Percy J.R. (ed.), The Study of Variable Stars Using Small Telescopes. Cambridge University Press, 165

Sterken C., 1993, in (eds.) Kilkenny D., et al., Precision photometry, SAAO, 63

Sterken C., Manfroid J., Anton K., et al., 1993, Second Catalogue of Stars Measured in the Long-Term Photometry of Variables Project (1986-1990), ESO Sci. Rep. No. 12

Sterken C., Manfroid J., Beele D., et al., 1995a, Fourth Catalogue of Stars Measured in the Long-Term Photometry of Variables Project (1992-1994), ESO Sci. Rep. No. 16

Sterken C., Manfroid J., Beele D., et al., 1995b, A\&AS 113, 31

St-Louis N., Howarth I.D., Willis A.J., et al., 1993, A\&A 267, 447

St-Louis N., 1994, Ap. Sp. Sc. 221, 197

St-Louis N., Dalton M.J., Marchenko S.V., et al., 1995, ApJ 452, L57

Underhill A.B., Yang S., 1991, ApJ 368, 588

White R.L., Long K.S., 1986, ApJ 310, 832 


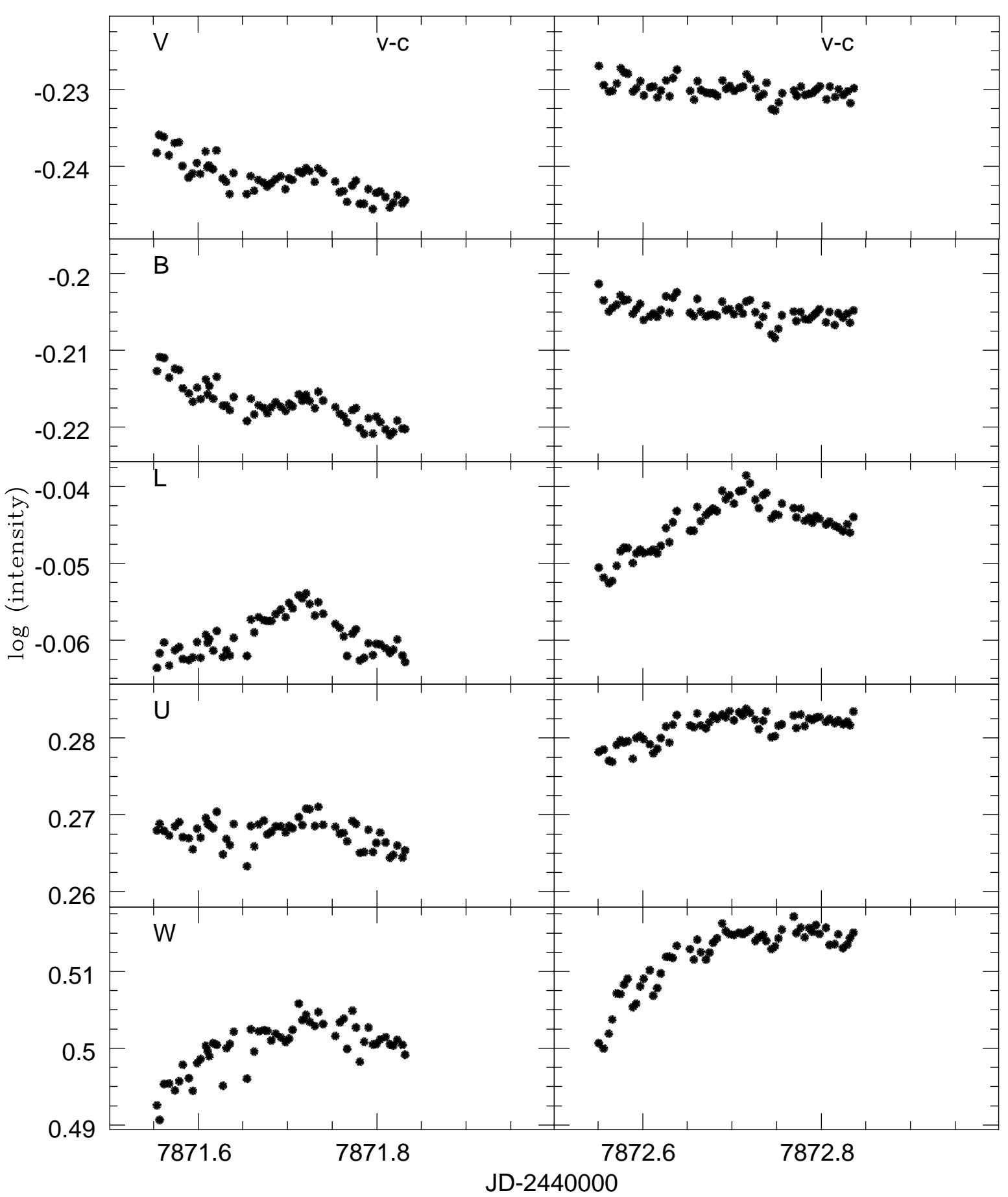

Fig. 9. Differential Walraven $V, B, L, U$ and $W$ on December 11, 1989 and December 12, 1989 for EZ CMa. 0.01 in log(intensity) scale $=0.025$ 


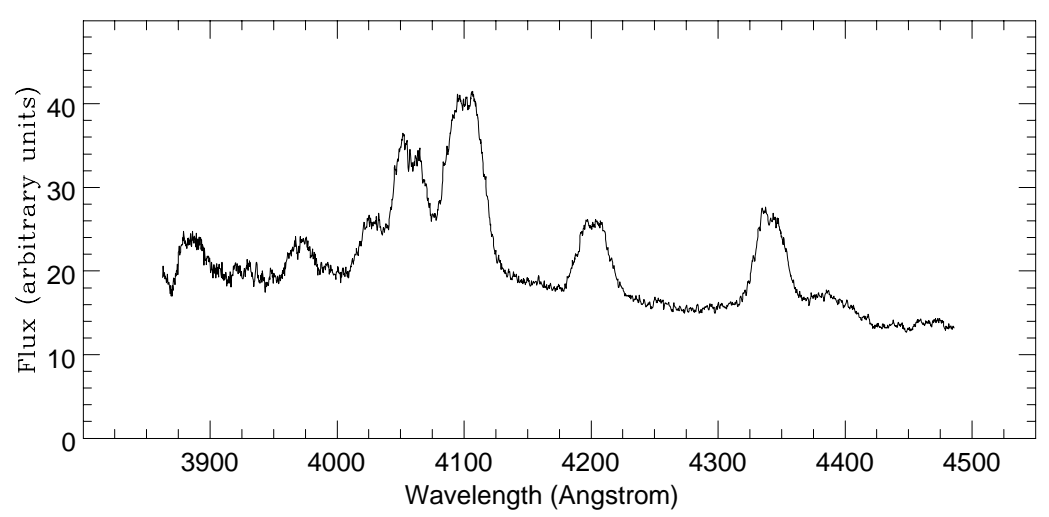

Fig. 10. Spectrum of EZ CMa taken at 4:32 UT on December 5, 1986 with an integration time of $10^{\mathrm{m}} 50^{\mathrm{s}}$

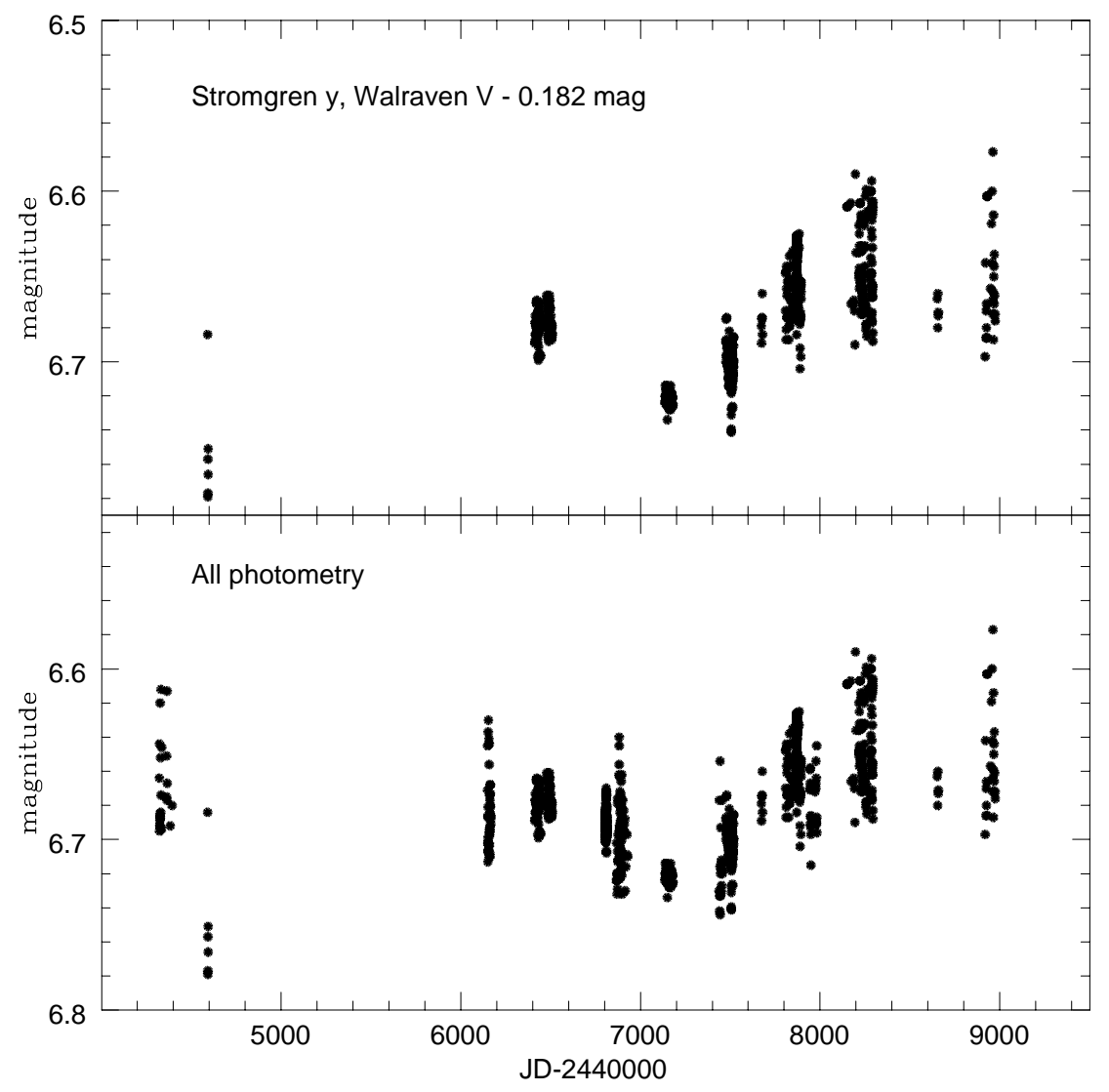

Fig. 11. Strömgren $y$, corrected Walraven $V$ and corrected Johnson $V$ photometry as a function of time, clearly showing a long time scale variation 


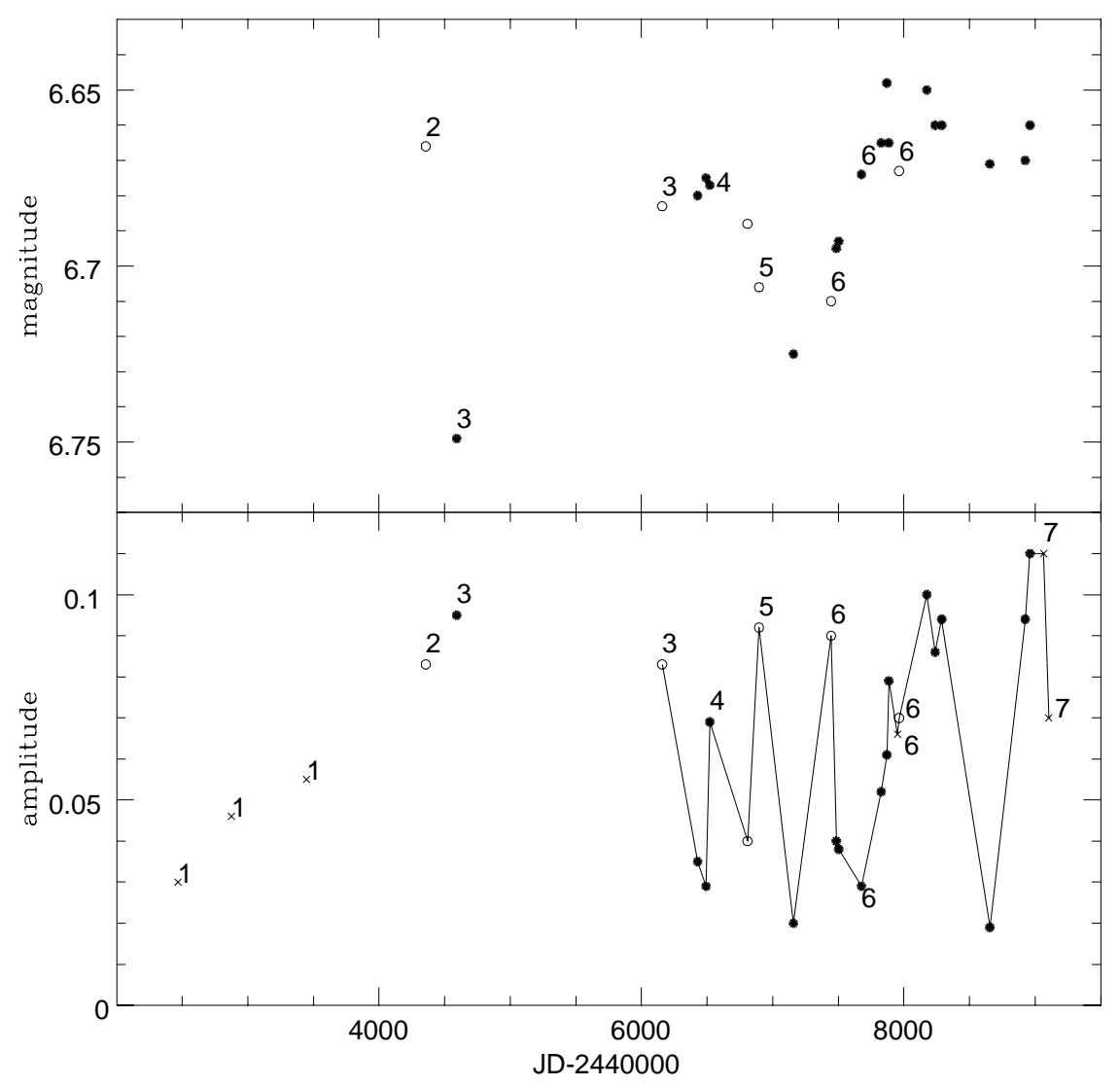

Fig. 12. Average magnitude and amplitude of the light curves over a period of 18 years, filled dots are Strömgren $y$ and corrected Walraven $V$ observations, open dots are corrected Johnson $V$ measurements, crosses are other filters. 1) Firmani et al. (1980); 2) Cherepashchuk (1981); 3) Lamontagne et al. (1986); 4) van Genderen et al. (1987); 5) Drissen et al. (1989); 6) Robert et al. (1992); and 7) Antokhin et al. (1994), rest: this paper

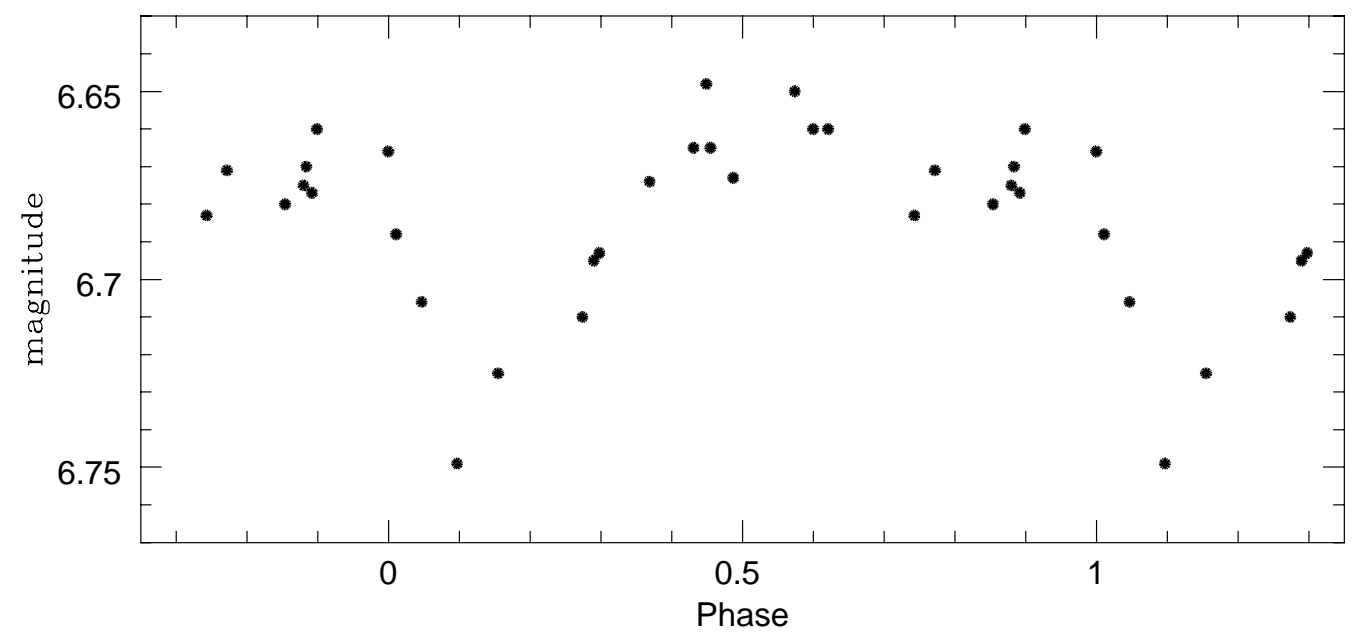

Fig. 13. The phase diagram for the average $y$ magnitude per data set for a period of $2425^{\mathrm{d}}(6.6 \mathrm{y})$ 


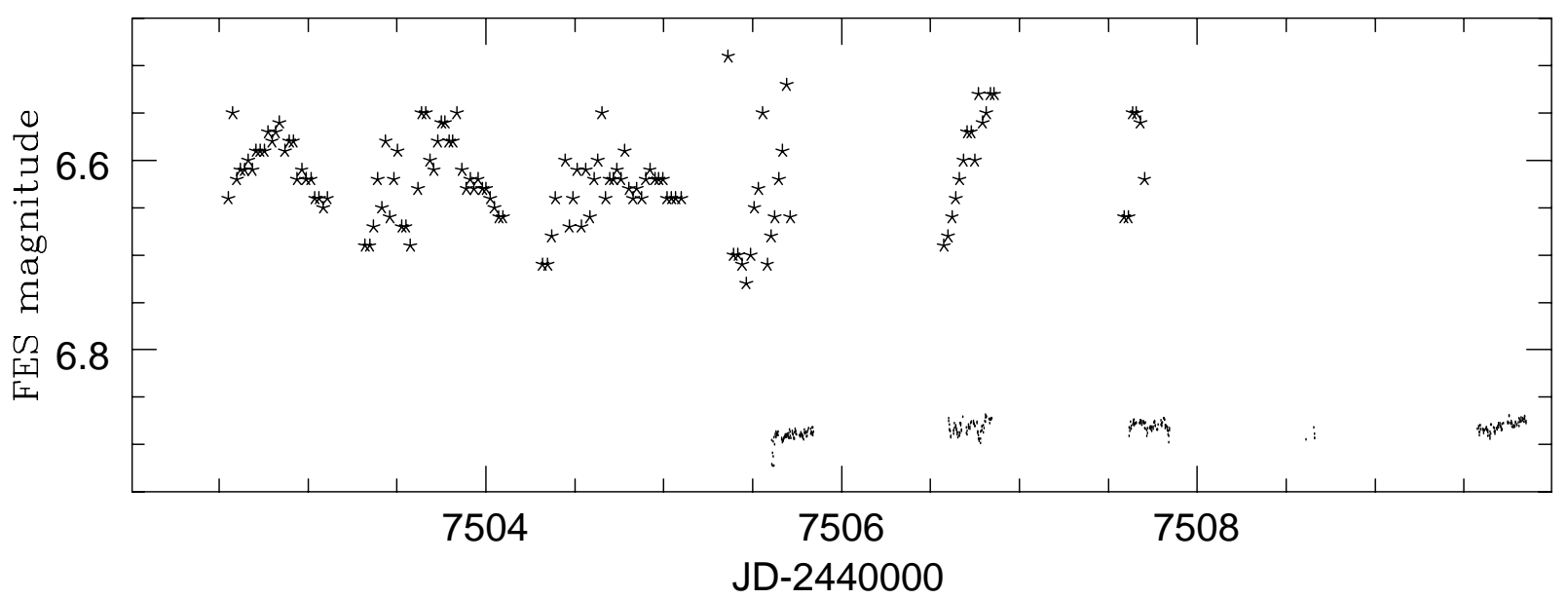

Fig. 14. Comparison of our observations from JD 2.447.505 through JD 2.447.510 ( $V_{\mathrm{J}}$ transformed from $V$ without correction of $\left.-0 .{ }^{\mathrm{m}} 182\right)$ and the FES magnitudes of St-Louis et al. (1993)

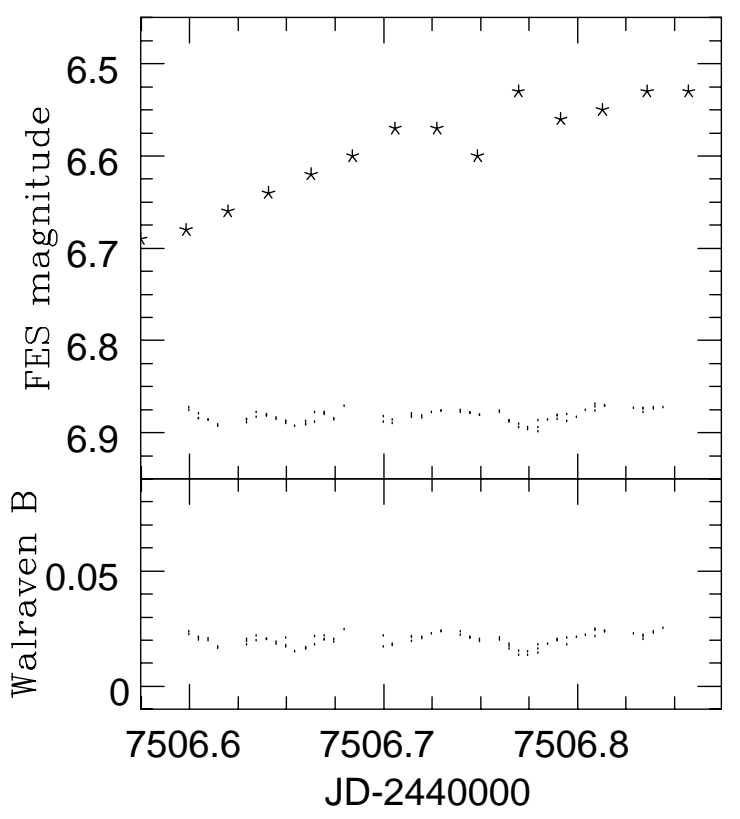

Fig. 15. More detailed Walraven $V$ and $B$ and FES light curves from JD 2.447.06 

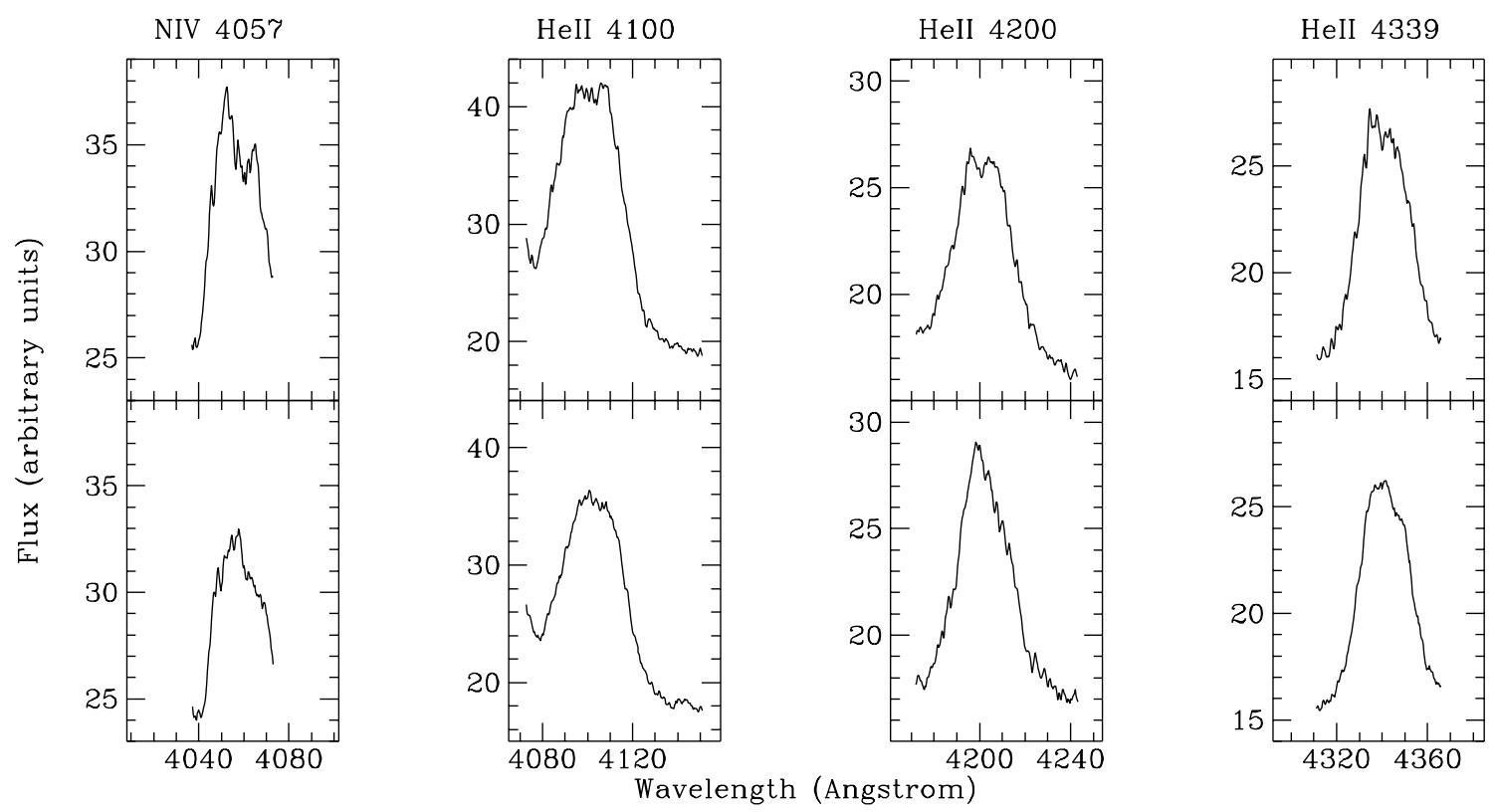

Fig. 16. Averaged line profiles of four major lines in the spectrum of EZ CMa, upper profile is averaged profile of December 5 , 1986, lower profile is averaged profile of December 7, 1986
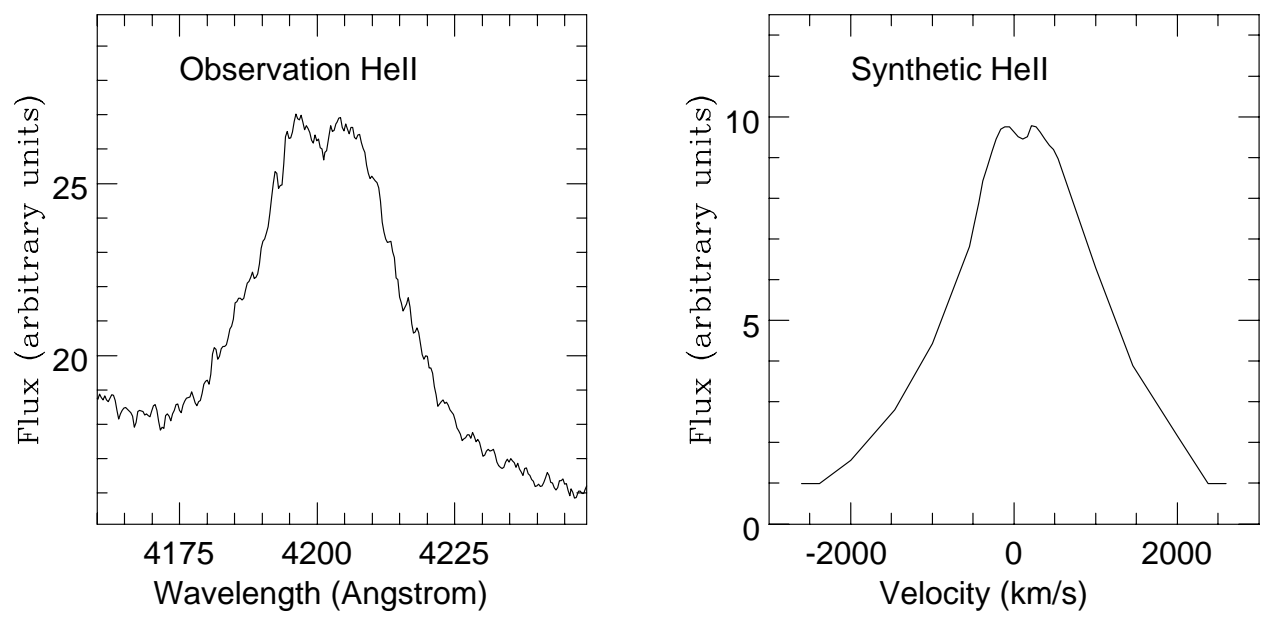

Fig. 17. Comparison of an observation of the HeI line at $4200 \AA$ with a synthetic HeI line profile of Koenigsberger (1995) 
Table 2. Photometric data of the comparison stars calibrated in the uvby, the $U B V$ and the $V B L U W$ system and their standard deviations

\begin{tabular}{|c|c|c|c|c|c|}
\hline \multirow[t]{2}{*}{ Star } & \multicolumn{4}{|c|}{ Strömgren system } & \\
\hline & $u$ & $v$ & $b$ & $y$ & \\
\hline \multirow[t]{2}{*}{ HD 50853} & 7.638 & 6.385 & 6.237 & 6.235 & \\
\hline & \pm 0.003 & \pm 0.002 & \pm 0.002 & \pm 0.005 & \\
\hline \multirow{2}{*}{ HD 50711} & 9.181 & 7.839 & 7.545 & 7.444 & \\
\hline & \pm 0.003 & \pm 0.003 & \pm 0.003 & \pm 0.005 & \\
\hline \multirow[t]{4}{*}{ HD 50806} & 8.003 & 6.780 & 6.566 & 6.533 & \\
\hline & \pm 0.001 & \pm 0.001 & \pm 0.001 & \pm 0.002 & \\
\hline & \multicolumn{3}{|c|}{ Johnson system } & & \\
\hline & $U$ & $B$ & $V$ & & \\
\hline \multirow[t]{4}{*}{ HD 51733} & 5.855 & 5.826 & 5.463 & & \\
\hline & \pm 0.014 & \pm 0.013 & \pm 0.010 & & \\
\hline & \multicolumn{5}{|c|}{ Walraven system } \\
\hline & $V$ & $B$ & $L$ & $U$ & $W$ \\
\hline \multirow[t]{2}{*}{ HD 50853 (I) } & 0.2561 & 0.2526 & 0.0919 & -0.1765 & -0.2838 \\
\hline & \pm 0.0084 & \pm 0.0035 & \pm 0.0123 & \pm 0.0105 & \pm 0.0043 \\
\hline \multirow[t]{2}{*}{ HD 50853 (II) } & 0.2581 & 0.2530 & 0.0972 & -0.1783 & -0.2855 \\
\hline & \pm 0.0075 & \pm 0.0018 & \pm 0.0040 & \pm 0.0036 & \pm 0.0046 \\
\hline
\end{tabular}

Table 3. List of IDS spectra. Spectra 1 through 33 concern the night of December 5, 1986, spectra 34 through 44 concern the night of December 7, 1986

\begin{tabular}{|c|c|c|c|c|c|}
\hline No. & Start (UT) & Duration (s) & No. & Start (UT) & Duration (s) \\
\hline 1 & $01^{\mathrm{h}} 40^{\mathrm{m}} 42^{\mathrm{s}}$ & 867 & 23 & $06^{\mathrm{h}} 17^{\mathrm{m}} 12^{\mathrm{s}}$ & 641 \\
\hline 2 & $01^{\mathrm{h}} 58^{\mathrm{m}} 49^{\mathrm{s}}$ & 652 & 24 & $06^{\mathrm{h}} 30^{\mathrm{m}} 02^{\mathrm{s}}$ & 640 \\
\hline 3 & $02^{\mathrm{h}} 10^{\mathrm{m}} 01^{\mathrm{s}}$ & 657 & 25 & $06^{\mathrm{h}} 41^{\mathrm{m}} 01^{\mathrm{s}}$ & 650 \\
\hline 4 & $02^{\mathrm{h}} 23^{\mathrm{m}} 30^{\mathrm{s}}$ & 662 & 26 & $06^{\mathrm{h}} 51^{\mathrm{m}} 59^{\mathrm{s}}$ & 638 \\
\hline 5 & $02^{\mathrm{h}} 34^{\mathrm{m}} 43^{\mathrm{s}}$ & 657 & 27 & $07^{\mathrm{h}} 03^{\mathrm{m}} 03^{\mathrm{s}}$ & 647 \\
\hline 6 & $02^{\mathrm{h}} 45^{\mathrm{m}} 59^{\mathrm{s}}$ & 656 & 28 & $07^{\mathrm{h}} 16^{\mathrm{m}} 25^{\mathrm{s}}$ & 640 \\
\hline 7 & $03^{\mathrm{h}} 01^{\mathrm{m}} 52^{\mathrm{s}}$ & 650 & 29 & $07^{\mathrm{h}} 27^{\mathrm{m}} 38^{\mathrm{s}}$ & 643 \\
\hline 8 & $03^{\mathrm{h}} 12^{\mathrm{m}} 54^{\mathrm{s}}$ & 659 & 30 & $07^{\mathrm{h}} 38^{\mathrm{m}} 41^{\mathrm{s}}$ & 644 \\
\hline 9 & $03^{\mathrm{h}} 24^{\mathrm{m}} 11^{\mathrm{s}}$ & 664 & 31 & $07^{\mathrm{h}} 49^{\mathrm{m}} 46^{\mathrm{s}}$ & 637 \\
\hline 10 & $03^{\mathrm{h}} 35^{\mathrm{m}} 31^{\mathrm{s}}$ & 642 & 32 & $08^{\mathrm{h}} 03^{\mathrm{m}} 07^{\mathrm{s}}$ & 652 \\
\hline 11 & $03^{\mathrm{h}} 50^{\mathrm{m}} 41^{\mathrm{s}}$ & 651 & 33 & $08^{\mathrm{h}} 14^{\mathrm{m}} 16^{\mathrm{s}}$ & 653 \\
\hline 12 & $04^{\mathrm{h}} 01^{\mathrm{m}} 57^{\mathrm{s}}$ & 655 & 34 & $04^{\mathrm{h}} 55^{\mathrm{m}} 44^{\mathrm{s}}$ & 683 \\
\hline 13 & $04^{\mathrm{h}} 13^{\mathrm{m}} 12^{\mathrm{s}}$ & 647 & 35 & $05^{\mathrm{h}} 07^{\mathrm{m}} 12^{\mathrm{s}}$ & 662 \\
\hline 14 & $04^{\mathrm{h}} 24^{\mathrm{m}} 11^{\mathrm{s}}$ & 318 & 36 & $05^{\mathrm{h}} 18^{\mathrm{m}} 24^{\mathrm{s}}$ & 655 \\
\hline 15 & $04^{\mathrm{h}} 32^{\mathrm{m}} 09^{\mathrm{s}}$ & 650 & 37 & $05^{\mathrm{h}} 33^{\mathrm{m}} 26^{\mathrm{s}}$ & 735 \\
\hline 16 & $04^{\mathrm{h}} 43^{\mathrm{m}} 18^{\mathrm{s}}$ & 705 & 38 & $05^{\mathrm{h}} 45^{\mathrm{m}} 57^{\mathrm{s}}$ & 650 \\
\hline 17 & $04^{\mathrm{h}} 55^{\mathrm{m}} 24^{\mathrm{s}}$ & 661 & 39 & $05^{\mathrm{h}} 57^{\mathrm{m}} 03^{\mathrm{s}}$ & 649 \\
\hline 18 & $05^{\mathrm{h}} 06^{\mathrm{m}} 38^{\mathrm{s}}$ & 646 & 40 & $06^{\mathrm{h}} 09^{\mathrm{m}} 56^{\mathrm{s}}$ & 644 \\
\hline 19 & $05^{\mathrm{h}} 18^{\mathrm{m}} 21^{\mathrm{s}}$ & 660 & 41 & $06^{\mathrm{h}} 20^{\mathrm{m}} 55^{\mathrm{s}}$ & 1371 \\
\hline 20 & $05^{\mathrm{h}} 41^{\mathrm{m}} 32^{\mathrm{s}}$ & 739 & 42 & $06^{\mathrm{h}} 46^{\mathrm{m}} 58^{\mathrm{s}}$ & 681 \\
\hline 21 & $05^{\mathrm{h}} 54^{\mathrm{m}} 15^{\mathrm{s}}$ & 659 & 43 & $06^{\mathrm{h}} 58^{\mathrm{m}} 53^{\mathrm{s}}$ & 660 \\
\hline 22 & $06^{\mathrm{h}} 06^{\mathrm{m}} 16^{\mathrm{s}}$ & 636 & 44 & $07^{\mathrm{h}} 10^{\mathrm{m}} 29^{\mathrm{s}}$ & 638 \\
\hline
\end{tabular}


Table 4. List of all observations of EZ CMa over a period of 18 years, average magnitudes per data set: $y, V($ uncorrected), $V_{\mathrm{J}}$ (uncorrected) and the maximum light amplitude

\begin{tabular}{|c|c|c|c|c|c|c|}
\hline \multicolumn{2}{|c|}{ J.D.-2440000 } & No. of obs. & Magnitude & Amplitude & Filter & Last reference of discussion \\
\hline$\overline{2447}$ & 2484 & 80 & 7.013 & 0.030 & $5640 \AA$ & Firmani et al. 1980 \\
\hline 2857 & 2892 & 22 & 6.993 & 0.046 & $5640 \AA$ & Firmani et al. 1980 \\
\hline 3441 & - $\quad 3454$ & 9 & 6.948 & 0.055 & $5200 \AA$ & Firmani et al. 1980 \\
\hline 4321 & - 4392 & 23 & 6.848 & 0.083 & Johnson $V$ & Cherepashchuk 1981 \\
\hline 4590 & - 4593 & 6 & 6.749 & 0.095 & Stromgren $y$ & Lamontagne et al. 1986 \\
\hline 6149 & - $\quad 6166$ & 42 & 6.865 & 0.083 & Johnson $V$ & Lamontagne et al. 1986 \\
\hline 6412 & - 6443 & 51 & 6.680 & 0.035 & Stromgren $y$ & this study \\
\hline 6475 & - $\quad 6509$ & 35 & 6.675 & 0.029 & Stromgren $y$ & this study \\
\hline 6516 & - $\quad 6526$ & 10 & 6.859 & 0.069 & Walraven $V$ & v. Genderen et al. 1987 \\
\hline 6806 & - $\quad 6811$ & 281 & 6.870 & 0.040 & Johnson $V$ & this study \\
\hline 6865 & $\begin{array}{l}-\quad 6927\end{array}$ & 67 & 6.888 & 0.092 & Johnson $V$ & Drissen et al. 1989 \\
\hline 7135 & - 7180 & 57 & 6.725 & 0.020 & Stromgren $y$ & this study \\
\hline 7437 & - 7455 & 19 & 6.892 & 0.090 & Johnson $V$ & Robert et al. 1992 \\
\hline 7474 & - 7495 & 21 & 6.695 & 0.040 & Stromgren $y$ & this study \\
\hline 7490 & - $\quad 7512$ & 418 & 6.875 & 0.038 & Walraven $V$ & this study \\
\hline 7673 & - $\quad 7680$ & 6 & 6.674 & 0.029 & Stromgren $y$ & Robert et al. 1992 \\
\hline 7807 & - $\quad 7847$ & 30 & 6.665 & 0.052 & Stromgren $y$ & this study \\
\hline 7869 & - $\quad 7872$ & 218 & 6.830 & 0.061 & Walraven $V$ & this study \\
\hline 7875 & - $\quad 7894$ & 21 & 6.665 & 0.079 & Stromgren $y$ & this study \\
\hline 7930 & - $\quad 7971$ & 32 & 6.785 & 0.066 & $5388 \AA$ & Robert et al. 1992 \\
\hline 7944 & - $\quad 7983$ & 22 & 6.855 & 0.070 & Johnson $V$ & Robert et al. 1992 \\
\hline 8150 & - $\quad 8200$ & 10 & 6.650 & 0.100 & Stromgren $y$ & this study \\
\hline 8215 & - 8260 & 53 & 6.660 & 0.086 & Stromgren $y$ & this study \\
\hline 8281 & - 8294 & 39 & 6.660 & 0.094 & Stromgren $y$ & this study \\
\hline 8651 & $-\quad 8658$ & 5 & 6.671 & 0.019 & Stromgren $y$ & this study \\
\hline 8918 & - 8931 & 9 & 6.670 & 0.094 & Stromgren $y$ & this study \\
\hline 8948 & - 8974 & 17 & 6.660 & 0.110 & Stromgren $y$ & this study \\
\hline 9038 & - $\quad 9089$ & 154 & 6.995 & 0.110 & $5140 \AA$ & Antokhin et al. 1994 \\
\hline 9095 & - $\quad 9121$ & 19 & 6.980 & 0.070 & $5140 \AA$ & Antokhin et al. 1994 \\
\hline
\end{tabular}

Table 5. Line emission contribution to the total flux in the three filter systems

\begin{tabular}{llll}
\hline Filter system & Filter & $\begin{array}{l}\lambda_{\text {eff }} \\
\AA\end{array}$ & $\begin{array}{l}\text { Line emission contribution to the total flux } \\
\%\end{array}$ \\
\hline Strömgren & $u$ & 3505 & 26 \\
& $v$ & 4110 & 33 \\
& $b$ & 4685 & 67 \\
& $y$ & 5488 & 21 \\
\hline Johnson & $U$ & 3650 & 22 \\
& $B$ & 4400 & 30 \\
& $V$ & 5500 & 11 \\
\hline Walraven & $V$ & 5403 & 11 \\
& $B$ & 4280 & 22 \\
& $L$ & 3840 & 10 \\
& $U$ & 3618 & 16 \\
& $W$ & 3234 & 37 \\
\hline
\end{tabular}


Table 6. Identifications of the emission lines in the three filter systems. + means major line contribution, - minor line contribution. Note (a): the symmetry of this line changes considerably

\begin{tabular}{|c|c|c|c|c|c|c|c|c|c|c|c|c|}
\hline \multirow{2}{*}{ Contributing lines } & \multicolumn{4}{|c|}{ Strömgren filters } & \multicolumn{5}{|c|}{ Walraven filters } & \multicolumn{3}{|c|}{ Johnson filters } \\
\hline & $u$ & $v$ & $b$ & $y$ & $V$ & $B$ & $L$ & $U$ & $W$ & $U$ & $B$ & $V$ \\
\hline He i 3187.7 & & & & & & & & & - & - & & \\
\hline He II 3203.1 & & & & & & & & & + & + & & \\
\hline N IV 3478.7 & + & & & & & & & + & & + & & \\
\hline N IV 3483.0 & + & & & & & & & + & & + & & \\
\hline N IV 3485.0 & + & & & & & & & + & & + & & \\
\hline N IV 3689 & & & & & & & & - & & & & \\
\hline N IV 3696 & & & & & & & & - & & & & \\
\hline N IV 3714 & & & & & & & & - & & & & \\
\hline N III 3745.8 & & & & & & & - & - & & & & \\
\hline N IV 3747.5 & & & & & & & - & - & & & & \\
\hline N III 3754.6 & & & & & & & - & - & & & & \\
\hline N III 3771.1 & & & & & & & - & & & & & \\
\hline He II 3813.5 & & & & & & & - & & & & & \\
\hline He II 3833.8 & & & & & & & - & & & & & \\
\hline He II 3858.1 & & & & & & & - & & & & & \\
\hline He II 3887.4 & & & & & & & - & & & & & \\
\hline He I 3888.6 & & & & & & & - & & & & & \\
\hline He II 3923.5 & & & & & & & - & & & & & \\
\hline He II 3968.4 & & & & & & & - & & & & & \\
\hline He II 4025.6 & & - & & & & - & & & & & & \\
\hline $\bar{N}$ IV 4057.8 & & + & & & & - & & & & & - & \\
\hline Si IV 4088.9 & & - & & & & - & & & & & & \\
\hline N III 4097.3 & & + & & & & + & & & & & - & \\
\hline He II 4100.0 & & - & & & & - & & & & & & \\
\hline N III 4103.4 & & + & & & & + & & & & & - & \\
\hline Si IV 4116.1 & & - & & & & - & & & & & & \\
\hline He II 4199.8 & & + & & & & + & & & & & - & \\
\hline He II 4338.7 & & & & & & + & & & & & - & \\
\hline$\overline{\mathrm{N}}$ III 4379.1 & & & & & & - & & & & & & \\
\hline He II 4541.6 & & & & & & - & & & & & - & \\
\hline N V 4603.7 & & & - & & & - & & & & & - & \\
\hline N V 4620.0 & & & - & & & - & & & & & - & \\
\hline$\overline{\mathrm{N}}$ III 4634.2 & & & - & & & & & & & & - & \\
\hline N III 4640.6 & & & - & & & & & & & & - & \\
\hline N III 4641.9 & & & - & & & & & & & & - & \\
\hline He II $4685.7^{a}$ & & & + & & & - & & & & & + & \\
\hline He II 4859.3 & & & & & & & & & & & - & - \\
\hline He II 5411.5 & & & & + & + & & & & & & & + \\
\hline N II 5462.6 & & & & - & - & & & & & & & - \\
\hline N II 5480.1 & & & & - & - & & & & & & & - \\
\hline N II 5495.7 & & & & - & - & & & & & & & - \\
\hline C IV 5801.3 & & & & & - & & & & & & & - \\
\hline C IV 5812.0 & & & & & - & & & & & & & - \\
\hline He I 5875.6 & & & & & & & & & & & & - \\
\hline
\end{tabular}

